\title{
Space- and Time-Resolved Bolometry on ZT-4.0M
}

\author{
G. Miller \\ J. C. Ingraham \\ R. Cowan
}

\section{DISCLAIMER}

This report was prepared as an account of work sponsored by an agency of the United States Government. Neither the United States Government nor any agency thereof nor any of their employees, makes any warranty, express or implied, or assumes any legal liability or responsibility for the accuracy, completeness, or usefulness of any information, apparatus, product, or process disclosed, or represents that its use would not infringe privately owned rights. Reference herein to any specific commercial product, process, or service by trade name, trademark, manufacturer, or otherwise does not necessarily constitute or imply its endorsement, recommendation, or favoring by the United States Government or any agency thereof. The views and opinions of authors expressed herein do not necessarily state or reflect those of the United States Government or any agency thereof. 
SPACE- AND TIME-RESOLVED BOLOMETRY ON ZT-40M

by

G. Miller, J. C. Ingraham, and R. Cowan

\begin{abstract}
Progress in the development of a multichord bolometer system for the Los Alamos Reversed Field Pinch Program is reported. A six-channel system is now operating on ZT-40M. The bolometer element fabrication process and the data analysis are described and some preliminary experimental results presented.
\end{abstract}

\title{
I. INTRODUCTION
}

One of the basic quantitative measurements on a fusion plasma machine is the radiated power loss from the plasma. Since radiation processes are relatively well understood, it is of great interest to know whether or not radiation is mainly responsible for the plasma energy confinement time. The fraction of the total energy loss that occurs by radiation is thus a useful parameter of a fusion plasma experiment. Whether or not radiation is the doninant loss mechanism, the measured radiated power provides a constraint in determining the impurity content of the plasma.

Radiation loss measurements are now being done quite routinely on Tokamaks and Stellarators. This however is not the case for the reversed field pinch and other machines which might generally be described as having shorter time scales and more electrically noisy plasmas. During the past five years we have been using two single channel systems on ZT-40M: a bolometer employing $5 \mathrm{MHz}$ excitation of the resistor, 1 and an infrared calorimeter. ${ }^{2}$ These single channel measurements were used to infer the radiative loss fraction. Measurement of the radiative fraction has also been reported from HBTX-1A. ${ }^{3}$ However, it should be noted that when the energy detector is withdrawn far from the liner surface, as was the case particularly for HBTX, 
significant errors are possible depending upon whether the assumed source profile matches that actually present. Or this basis, tine multichord bolometer, described here, as well as our infrarad calorimeter system, which allowed the detection foil to be very close to the liner surface, provides more definitive measurements of the radiated power fraction.

The basic method, which is almost universally employed for absolutely calibrated total radiation power measurements, is the measurement of the temperature rise of a thin absorber. Possible systematic errors of this method are (1) incomplete absorption of the incident radiation, (2) reflection of the incident radiation, or (3) detection of nonradiative energy fluxes such as neutral particles. None of these possible problems is serious: (1) the relevant photon energy, say $20-200 \mathrm{eV}$, is very strongly absorbed and very thin absorbing layers are possibie; (2) reflection coefficients ${ }^{4}$ are also small; and (3) estimates* based on silicon probe measurements, time-of-flight spectrometer measurements, and neutral particle transport simulations imply that the energy loss by neutral particles is small; furthermore, the reflection scefficient for low energy neutrals is high, reducing their detection efficiency. Regarding the issue of radiation reflection, although this is a small effect which is not significant at present levels of accuracy, reflections from the metal liner will also have to be taken into account.

Here we report on progress in the development of a multichord bolometer system for the reversed field pinch program. Our present single channel bolometer on ZT-4CM uses a bolometer element that consists of a 1-mil aluminum absorber with a thin, die-cut, platinum foil resistor epoxied to it. The gluing process was difficult and the results uncertain as to the thickness of the glue layer, which had a large effect on the time constant as well as some effect on the calibration factor. We have now developed a process using evaporative techniques and photolithography for producing boloneter elements more uniformly. This tiniformity is essential for a multichord application. We have again used a 1-mil aluminum absorber, having the advantage that most of the heat capacity is in the best characterized material (being almost entirely in the aluminum), but having the disadvantage of a somewhat low

\footnotetext{
‡ Information provided by T. Cayton, Los Alamos Natjonal I,aboratory Group CTR-6 (1985).
} 
sensitivity for a highly collimated application. Photos of the bolometer are shown in Figs. 1,2 and 3 .

At present a six channel system is working on $2 T-40 M$ (only six chords were unused by other diagnostics and available for bolometry). Some preliminary data are presented in this report, although radiation profile trends in important operating regimes have not yet been studied. In the next year we plan to upgrade the signal-to-noise ratio of the multichannel system by a factor of at least 3 , either through a lower heat capacity bolometer element or by improving the electronics, or both.

\section{BOLOMETER ELEMENT FABRICATION}

The bolometer elements consisted of three layers: a $25 \mu$-thick aluminum fcil, a $2 \mu$-thick parylene insulating layer, and a $0.3 \mu$-thick gold resistor. The aluminum foil was glued to $2^{\prime \prime}$-square $\times 1 / 16^{\prime \prime}$-thick glass plates used as a support for the foil during the fabrication procedure. The foil was placed between two sheets of paper and rolled with a photographic print roller to remove wrinkles prior to the gluing. In the gluing operation a sheet of Aremco Wafer-Mount 562 sheet glue ( $3 \mathrm{mils}$ thick) was placed between a piece cf aluminum foil and a glass plate. One-nil-thick Teflon was placed next to the glass and the foil to prevent sticking. A piece of stainless steel with a \#4 finish was placed on the foil side of the stack. This was done to help prevent the foil from wrinkling. Finally, squares of $1 / 8^{\prime \prime}$-thick aluminum were placed on the outside of the stack. C-clamps were used to hold tine assembly together. This assembly was placed in an oven at $100^{\circ} \mathrm{C}$ for 30 minutes. After disassembly, the aluminum foil was trimmed to the dimensions of the glass slide.

To promote adhesion of the Parylene film, a silane treatment was applied to the aluminum foil. This was done by immersion in a $0.1 \%$ solution of A-174 silane in methanol for 30 minutes and followed by drying, reimmersion in methanol for 30 seconds, and final drying. The Parylene coating ras applied in a Union Carbide Model 1030 deposition system. A dimer charge of 5 grams of Parylene C. was used.

The bolometer iesistor pattern was delineated on the aluminum foil by photolithography. KTI positive photoresist was spun on the aluminum foil at $4000 \mathrm{rpm}$ and then dried at $60^{\circ} \mathrm{C}$ for 30 minutes. A positive film photo master was held against the photoresist with a glass slide and exposure was made in a 


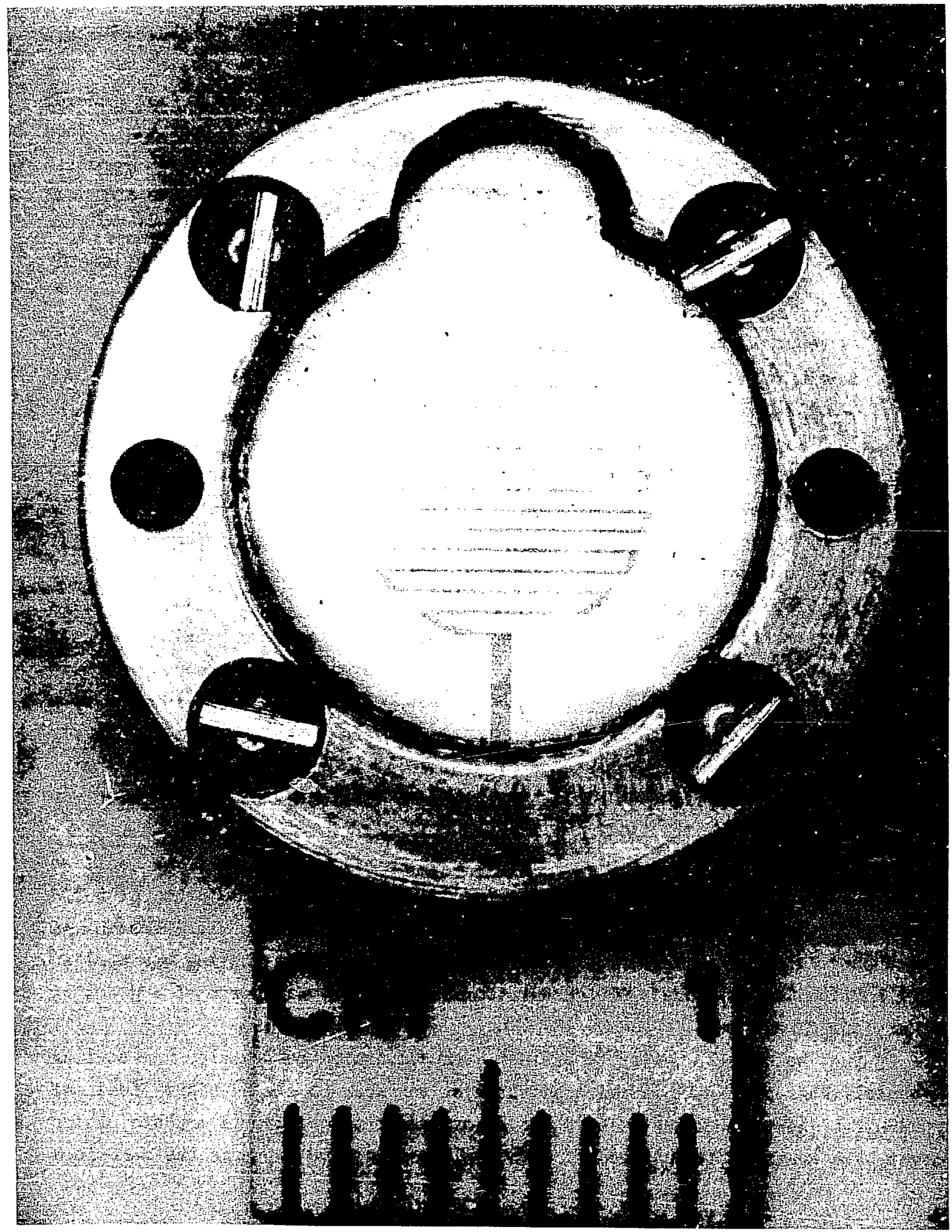

Fig. 1.

Bolometer resistor element (gold). 


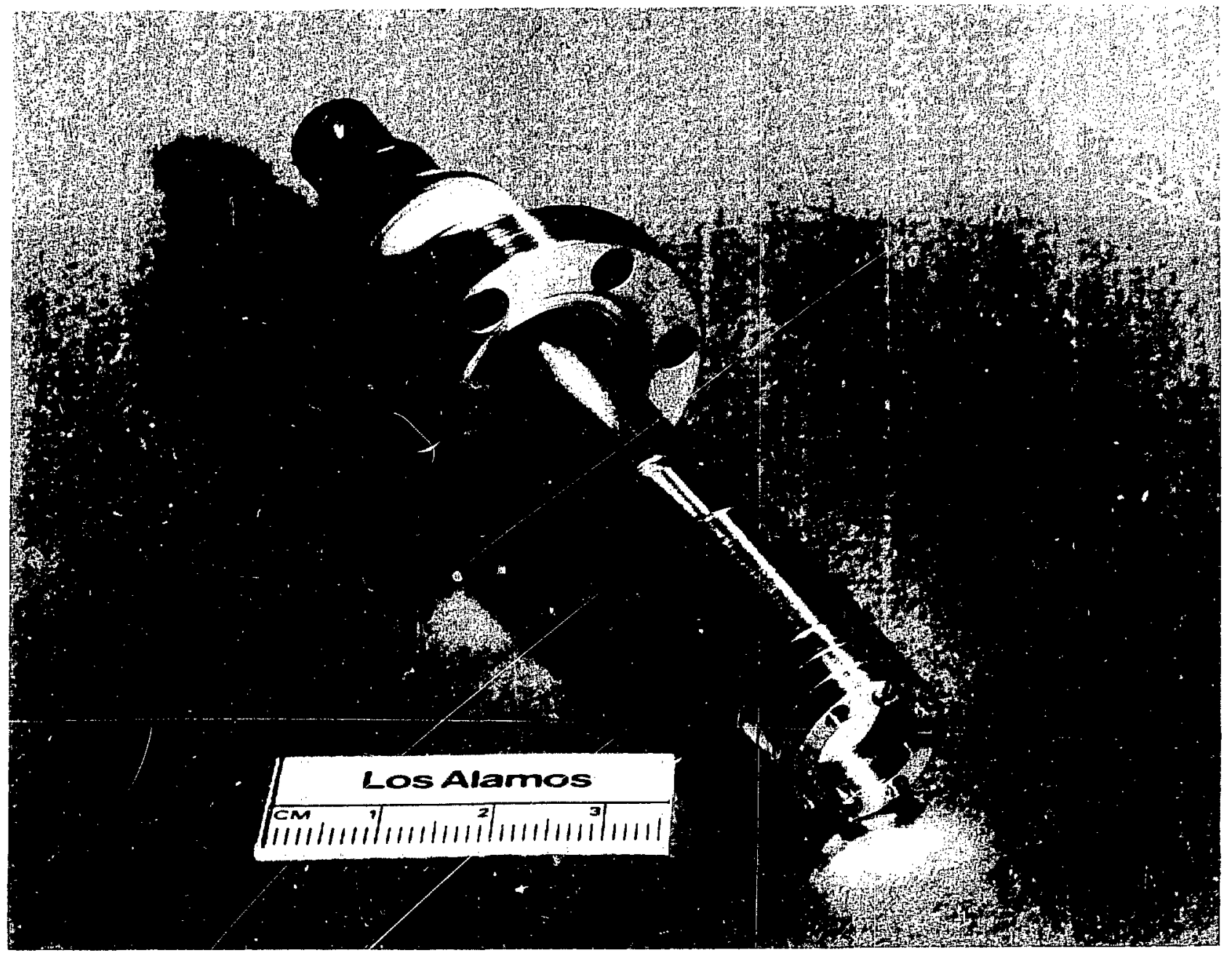

Fig. 2 .

View of the bolometer showing the aluminum foil absorber. 


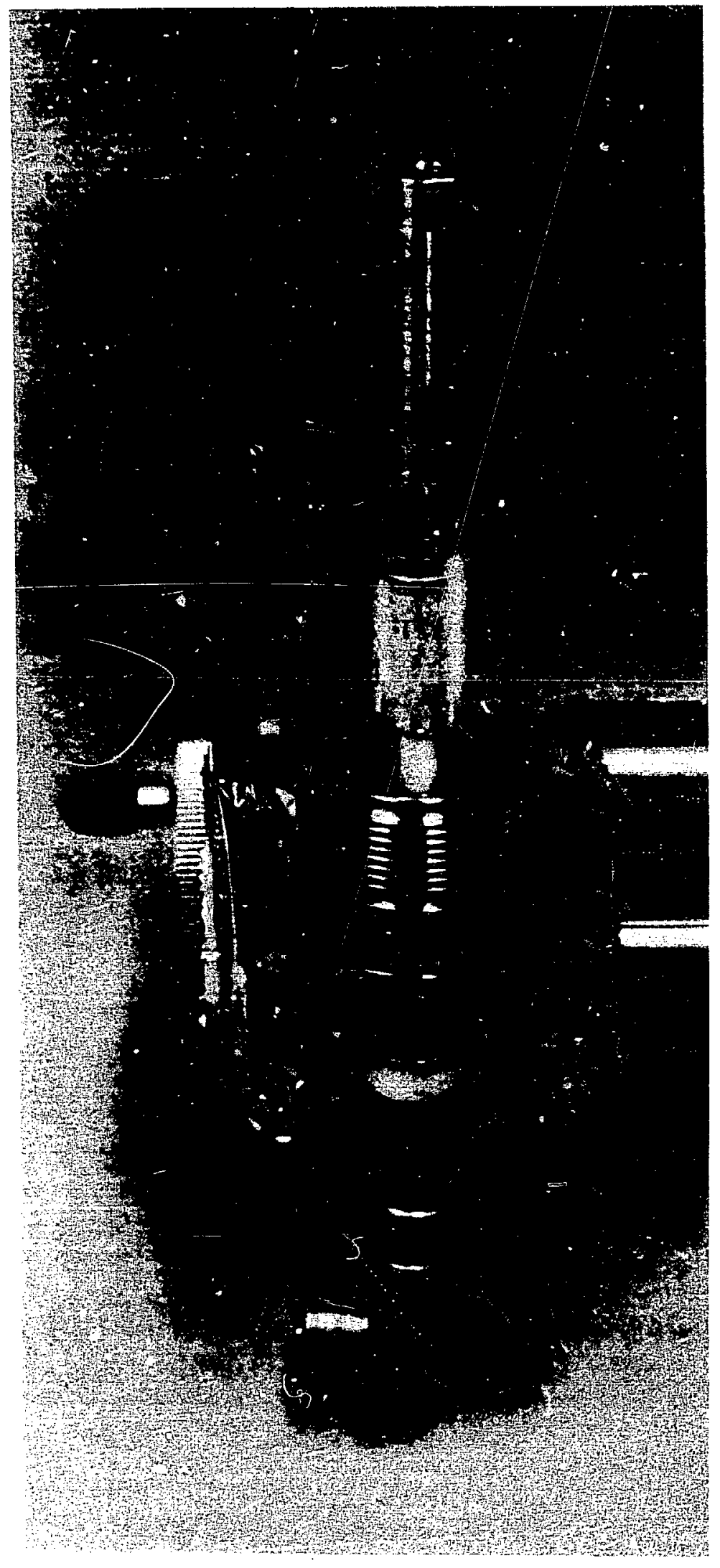

Fig. 3.

Bolometer with collimator attached. 
Kasper mask aligner for 20 seconds. The image was developed using Shipley positive photoresist developer. A one minute rinse in deionized water removed the excess developer.

Immediately before applying the gold, the samples were soaked for five minutes in chlorobenzene and blown dry with nitrogen. This step was necessary to promote adhesion of gold to the Parylene. The gold was applied to the surface using an electron-beam evaporator. The system was puinped to about $4 \times 10^{-7}$ torr and an evaporation rate of about $30 \AA / \mathrm{sec}$ was used. The deposition was controlled using an Inficon crystal monitor.

The photolithographic "lift" technique was used to form the final image. The samples were soaked in acetone to dissolve the photoresist. A 000 artist's brush was used to gently remove the gold from unwanted areas. This operation was carried out using a binocular microscope.

The final step involved removing the aluminum foil from the glass plate. This was done by heating on a hot plate and gently peeling the foil from the glass. The adhesive was removed from the foil by soaking in warm $\left(30^{\circ} \mathrm{C}\right)$ trichloroe thylene.

III. DATA ANALYSIS

The measured quantities are the temperature increases during the shot of the bolometers, $T_{i}(t), i=1, N$, with six channels at present. These temperature histories are assumed to be related to the incident radiation power $p_{i}$ by the equation (neglecting fast thermal transients)

$$
\frac{d T_{i}}{d t}=-\frac{T_{i}}{\tau}+\frac{p_{i}}{H A},
$$

where $\tau$ represents transverse heat loss of the bolometer foil to the support structure $(\tau=100 \mathrm{~ms}), \mathrm{H}$ is the total heat capacity per unit area of the bolometer, and $\mathrm{A}$ is the area. By design, the total heat capacity in ouc case is almost entirely that of the 1-mil-thick aluminum absorbing layer, so that

$$
\mathrm{H} \simeq \mathrm{Ct},
$$


where $\mathrm{C}$ is the heat capacity per unit volume of aluminum and $t$ is the thickness of the aluminum.

The object of the data analysis is to deduce from the detected powers $\mathbf{p}_{i}$ or energies $E_{i}=\int p_{i} d t$ determined using Eq. (1), the power radiated by the plasma (in $W / \mathrm{cm}^{3}$ ), which will be denoted by $e$. The power incident on bolometer $i$ is given by

$$
p_{i}=\int e d V \frac{d \Omega}{4 \pi}
$$

where the volume integral is taken over the entire plasma volume and $\mathrm{d} \Omega$ is the solid angle (possibly zero) subtended by the bolometer from the particular spatial point.

The fundamental simplifying assumption in the data analysis is that $e$ is not an arbitrary three-dimensional function of space, but a flux surface quantity so that

$$
e=e(f) \text {, }
$$

with $f$ a flux surface label (defined here so that $f=0$ is the magnetic axis and $\mathrm{f}=1$ is the outermost plasma flux surface). The integration coordinates used in Eq. (2) are taken to be the five quantities $\rho_{1}, \alpha_{1}, \rho_{2}, \alpha_{2}$ and $\mathrm{Z}$ defined by Fig. 4. In terms of Cartesian coordinates X, Y, Z defined by Fig. 5, the spatial point of the integration is

$$
\begin{aligned}
& X=X_{i}+\rho_{1} \cos \alpha_{1}+\frac{Z-Z_{1}}{Z_{2}-Z_{1}}\left(\rho_{2} \cos \alpha_{2}-\rho_{1} \cos \alpha_{1}\right) \\
& Y=\rho_{1} \sin \alpha_{1}+\frac{Z-Z_{1}}{Z_{2}-Z_{1}}\left(\rho_{2} \sin \alpha_{2}-\rho_{1} \sin \alpha_{1}\right) \\
& Z=Z
\end{aligned}
$$




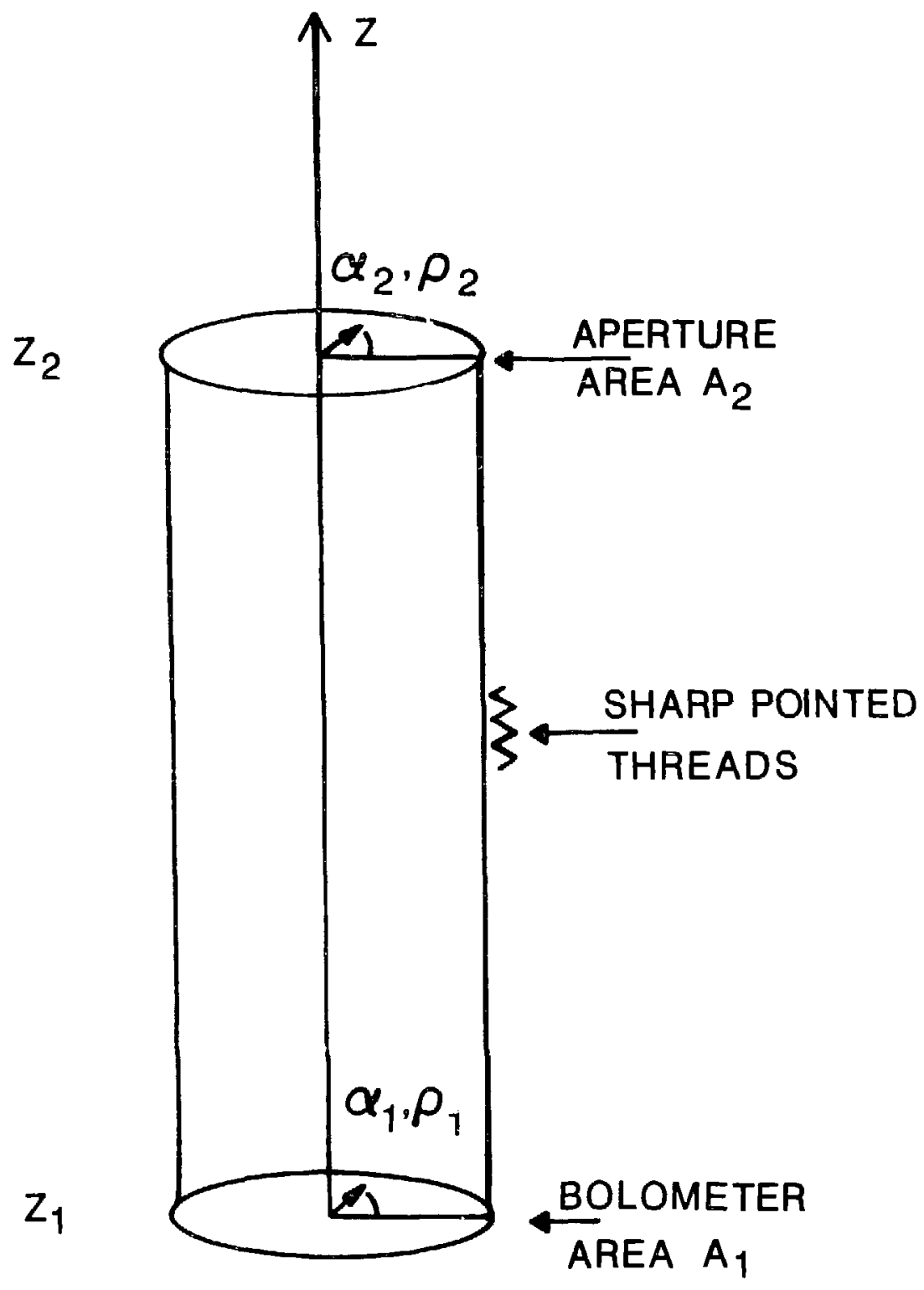

Fig. 4.

Coordinates used for acceptance integral, and defining apertures. The connecting tube has a threaded wall to limit reflections. 


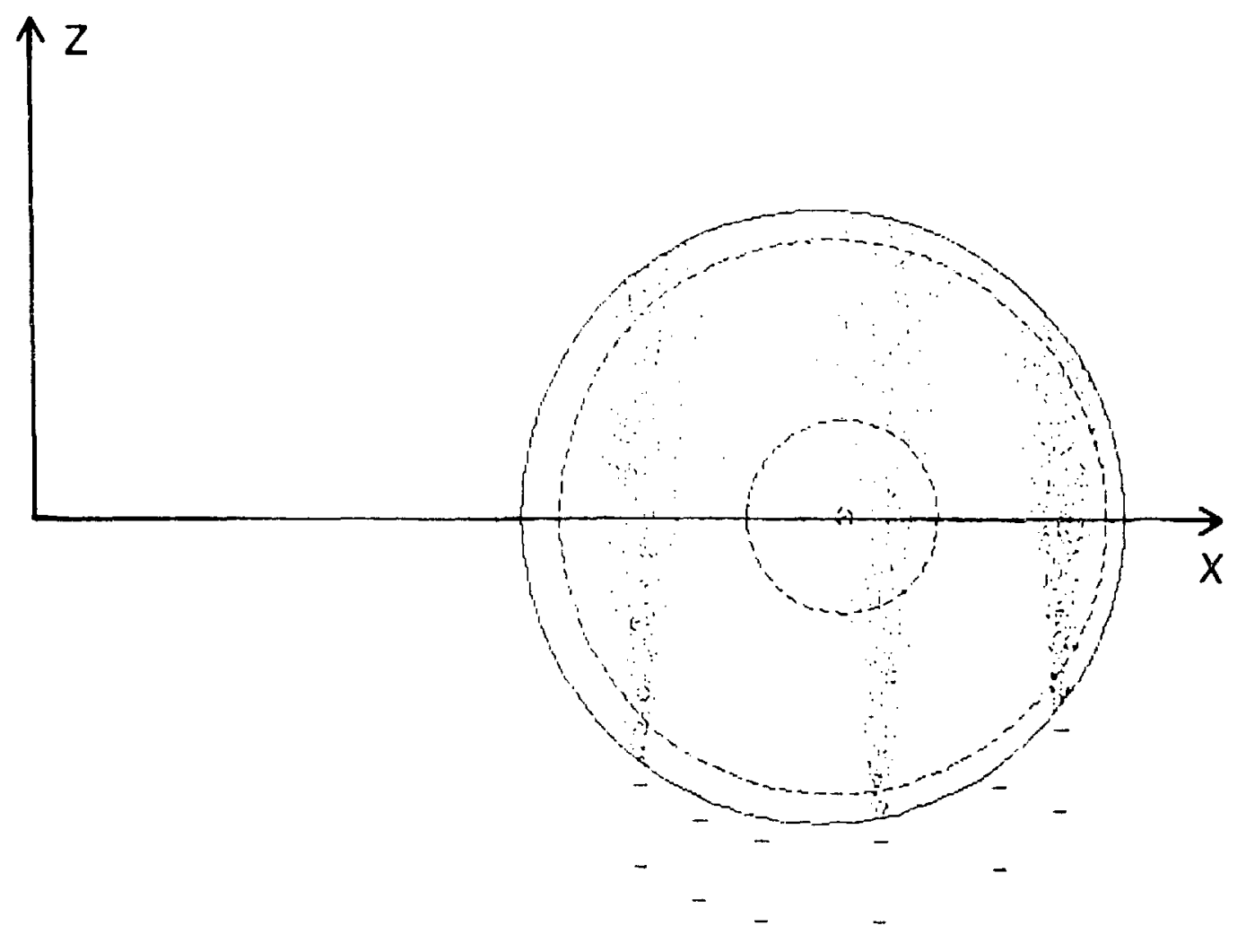

CHORD: $\begin{array}{llllll} & 1 & 2 & 3 & 5 & 7\end{array}$

Fig. 5 .

Cartesian coordinate system $X, Y$ (not shown) and $Z$. The multichord array is located at $Y=0$. The major axis of the torus is $X=0$. The dots show randomly located points occurring in the Monte Carlo integration. For clarity only three chords are shown. 
where $x_{i}$ is the $x$ position of the $i^{\text {th }}$ chord. Going now to a curved Cartesian system $x, y, z$ with $z$ along the minor axis of the torus,

$$
\begin{aligned}
& x=R_{0}-\left(X^{2}+Y^{2}\right)^{1 / 2} \\
& y=z,
\end{aligned}
$$

where $R_{0}$ is the major radius of the torus.

Finally $\mathrm{f} i \mathrm{~s}$ determined by the equation

$$
f=\frac{\left[[x-\xi(f)]^{2}+y^{2}\right\}^{1 / 2}}{{ }^{r} p},
$$

which must be solved iteratively for $f$. In $E q .(6), \xi(f)$ is the outward shift of the flux surface as a function of $f$, which must be specified. We assume a three parameter representation of the form

$$
\xi=\xi_{0}-\Delta \xi f^{\alpha}
$$

where $\xi_{0}$ is the on-axis shift, $\xi_{0}-\Delta \xi$ is the outside shift, and $\alpha$ is a power. From equilibrium modeling, ${ }^{5}$ parameter vaiues were chosen to be $\xi_{0}=0.08 \mathrm{r}_{\mathrm{p}}$, $\Delta \xi=0.044 \mathrm{r}_{\mathrm{p}}$, and $\alpha=2.7$. These values were tested by analyzing high accuracy 8-chord interferometer data and found to be optimum or nearly optimum. The quantity $r_{p}$ in $\mathrm{Eq} .(6)$ is the plasma radius taking into account scrape-off effects, so that

$$
r_{p}=r_{1}-\left(\xi_{0}-\Delta \xi\right)
$$

where $r_{l}$ is the effective outside limiter radius. 
The integration element of Eq. (2) is found to be

$$
d V \frac{d \Omega}{4 \pi}=\frac{d \rho_{1}^{2} d \alpha_{1} d \rho_{2}^{2} d \alpha_{2} d z}{16 \pi\left[\left(Z_{2}-Z_{1}\right)^{2}+\rho_{1}^{2}+\rho_{2}^{2}-2 \rho_{1} \rho_{2} \cos \left(\alpha_{1}-\alpha_{2}\right)\right]} .
$$

In the limit where the chords are well collimated, and the sideways shift is small, Eq. (2) reduces to

$$
p_{i}=\frac{A_{1} A_{2}}{4 \pi\left(Z_{2}-Z_{1}\right)^{2}} \int e(r) d z
$$

where $r$ is the minor radius, $A_{1}$ and $A_{2}$ are the areas of apertures at $z_{1}$ and $\mathrm{Z}_{2}$, and $\mathrm{Z}$ is a path length coordinate across the plasma. Aithough Eq. (10) is a useful first approximation, the analysis procedure is no more complicated including all known (or supposed; geometrical effects exactly, thereby limiting the approximation invoived in the data analysis to representation of e(f) by a few parameter functions. The noninear geometrical parameters involved in specifying flux surface shift are normally not varied but fixed at some reasonable values, so the data analysis consists of inverting the linear relation between the $N$ measurement quantities $p_{i}$ and a smaller number $n$ of parameters specifying $e(f)$. Such an Abel-like inversion process rapidly amplifies experimental errors as the number of parameters is increased, so we have decided initially to use $n=2$ so that $e(f)=C_{1} e_{1}(f)+C_{2} e_{2}(f)$. The choice of basis functions $e_{1}$ and $e_{2}$ is ultimately arbitrary and shown in Fig. 6. This choice of parameterization is such that a flat profile $\left(C_{1}=\omega_{2}\right)$ has approximately equal power loss from $e_{1}$ and $e_{2}$.

The linear relation between $\mathrm{C}_{k}{ }^{\prime} \mathrm{s}$ and $\mathrm{p}_{\mathrm{i}}{ }^{\prime} \mathrm{s}$ is denoted by

$$
\mathrm{p}_{\mathrm{i}}=\sum_{k=1}^{n} A_{i k} C_{k}
$$

The coefficients $A_{i k}$ are obtained by performing the integration in Eq. (2) for 


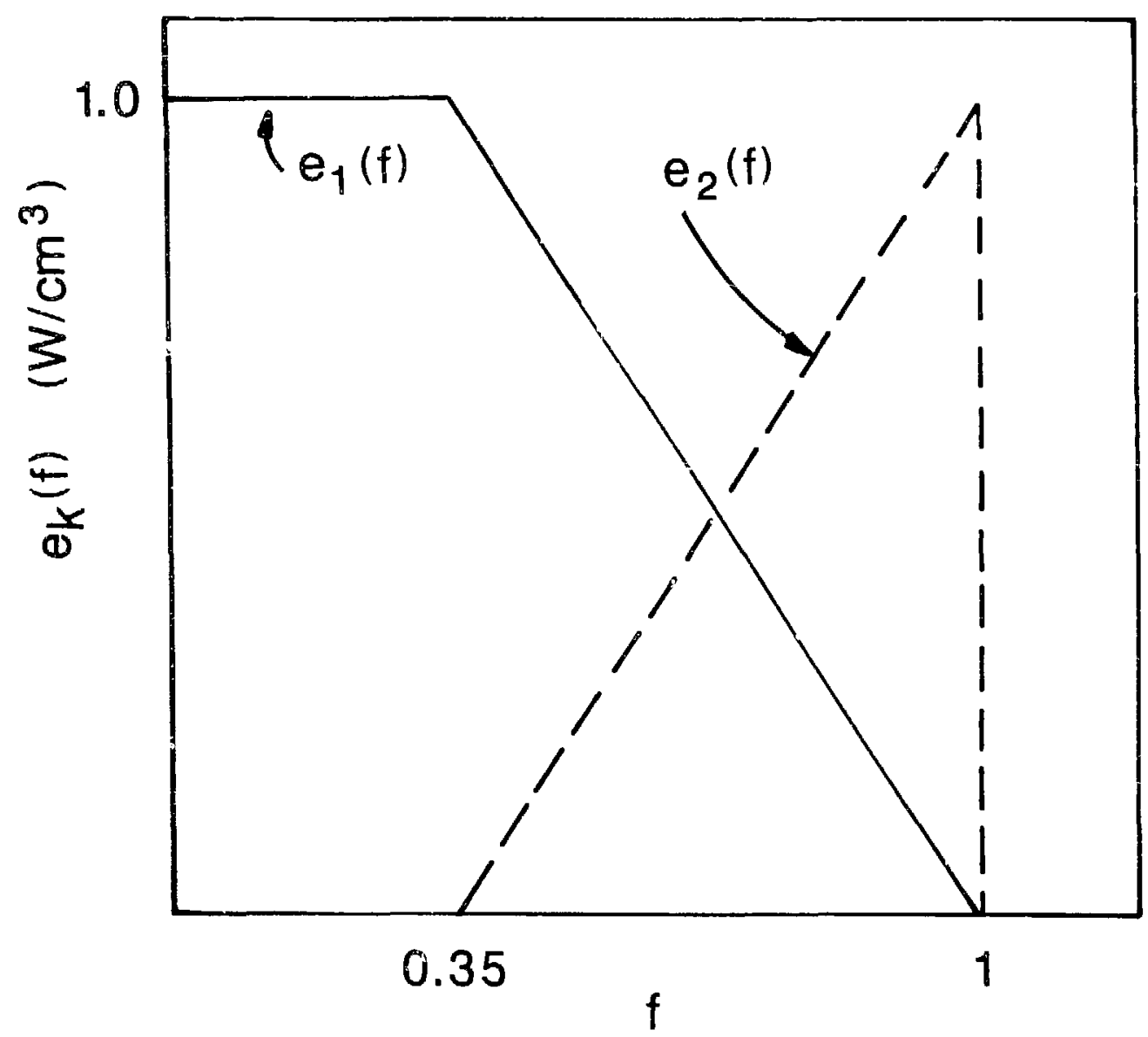

Fig. 6.

Definition of the radiation basis functions. 
$e(f)=e_{k}(f)$. The integration was done using a Monte Carlo technique illustrated in Fig. 5. Numerical values of $A_{i k}$ are shown in Fig. 7. By least-squares inverting the matrix $A$, we obtain the relation used to analyze the data, namely

$$
C_{k}=\left(A^{-1}\right)_{k i} P_{i}
$$

There are instances when the basic assumption of flux surface symmetry of the radiation [Eq. (3)] is observably not true, for example early time radiation from the inside wall of the torus probably associated with breakdown. Thus one must check that the fit to data obtained from Eqs. (11) and (12) is indeed adequate (in terms of $x^{2}$ ).

The total power radiated can be attributed partly to $e_{1}$ and partly to $e_{2}$, and thus divided up, similarly for the total energy since the matrix $A^{-1}$ is constant in time because we are not allowing the flux surface geometry to vary with time. The total energy radiated is then

$$
E_{r}=E_{r 1}+E_{r 2}
$$

with

$$
E_{r k}=P_{k} \sum_{i=1}^{N}\left(A^{-1}\right)_{k i} E_{i},
$$

where $E_{i}$ is the energy detected on the $i^{\text {th }}$ bolometer, and $P_{k}$ is the total power radiated from $e_{k}$.

$$
\begin{aligned}
& \text { Numerical values of } A^{-1} \text { are as follows }\left(e_{k \max }=1 \mathrm{~W} / \mathrm{cm}^{3}\right): \\
& c_{1}=-16.9 \mathrm{p}_{1}+2.3 \mathrm{p}_{2}+23.2 \mathrm{p}_{3}+29.8 \mathrm{p}_{5}-4.8 \mathrm{p}_{7}-20 \mathrm{p}_{8} ; \\
& \mathrm{C}_{2}=26.1 \mathrm{p}_{1}+9.3 \mathrm{p}_{2}-10.1 \mathrm{p}_{3}-16.4 \mathrm{p}_{5}+15.1 \mathrm{p}_{7}+26.5 \mathrm{p}_{8}
\end{aligned}
$$

The total radiated powers from $e_{1}$ and $e_{2}$ are given by 


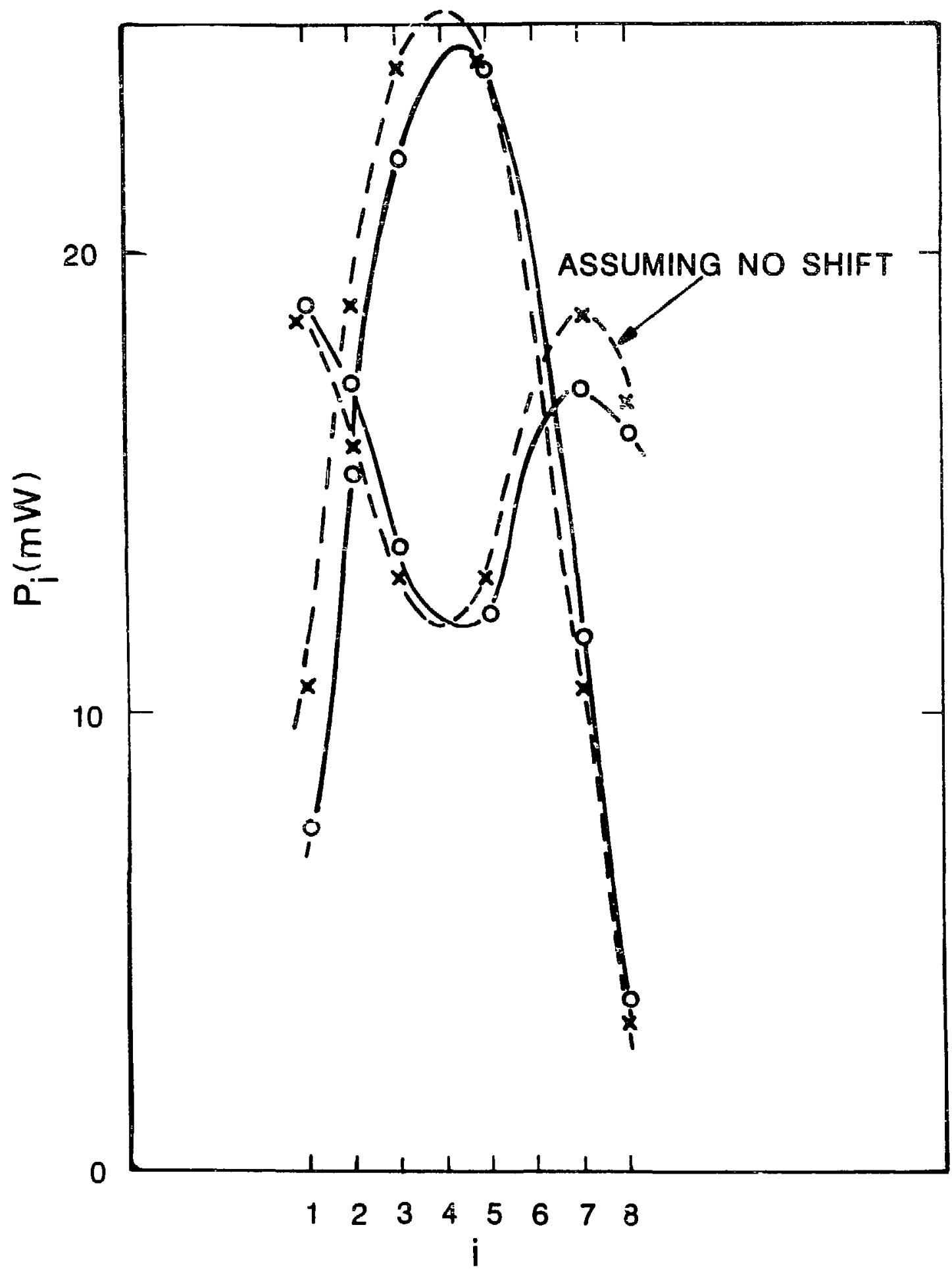

Fig. 7 .

Detected powers for the basis functions. The effect of sideways shifting of the flux surfaces is shown by calculating the case with no shift. 


$$
\begin{aligned}
& \mathrm{P}_{1}=0.40 \mathrm{MW}, \\
& \mathrm{P}_{2}=0.41 \mathrm{MW} .
\end{aligned}
$$

The total energy radiated, given by $E_{I}=P_{1} C_{1}+P_{2} C_{2}$ comes almost equally from each chord, as one would hope from the standpoint of error propagation. When $c_{1}=C_{2}=1$, the total radiated power is $0.81 \times 10^{6} \mathrm{~W}$, which is numerically equal to the total volume of the plasma in $\mathrm{cm}^{3}$.

IV. SOME EXPERIMENTAL RESULTS

Finally, in this section, some preliminary experimental data will be given, mostly to illustrate the quality of multichannel bolometer data now available on $\mathrm{ZT}-40 \mathrm{M}$. In Fig. $8 \mathrm{a}$ are shown measured (corrected for transverse cooling droop) temperature histories For a shot with a relatively large radiated power. Figure $8 \mathrm{~b}$ shows the same data on a long tine scale to illustrate the noise level. The noise in this case is mostly 60 cycle hum originating in the high gain circuitry of the FM receiver (for the multichannel bolometer, the FM receiver gain is 3 times greater than that for the single channel bolometer, which is described in Ref. 1). This noise could probably be eliminated.

Total radiated energy for this shot is shown in Fig. 9. The radiated energy is very nearly the total available for plasma losses, implying that the radiation losses are nearly 100\% in this case. The energy radiated centraily $\left(E_{r 1}\right)$ is $50 \%$ or greater of the total radiated energy $\left(E_{r}\right)$, which means that the radiation profile is flat or centrally peaked.

The more usual situation as far as the fraction of energy radiated is concerned is illustrated in Fig. 10. Note that in this case the radiation comes almost entirely from the plasma edge until about 8 ms.

our single channel bolometer gives a much better signal-to-noise ratio because of having about $35 x$ larger acceptance (although with a 20\% transmitting screen in front, giving a $7 \times$ larger temperature rise). However, the single channel boiometer, even though close to the plasma edge $(2 \mathrm{~cm})$ and therefore relatively insensitive to profiles, is nevertheless profile sensitive. For example, varying the assumed profile from moderately centrally peaked to edge peaked changes the inferred total radiated power by a factor of 1.9 . 


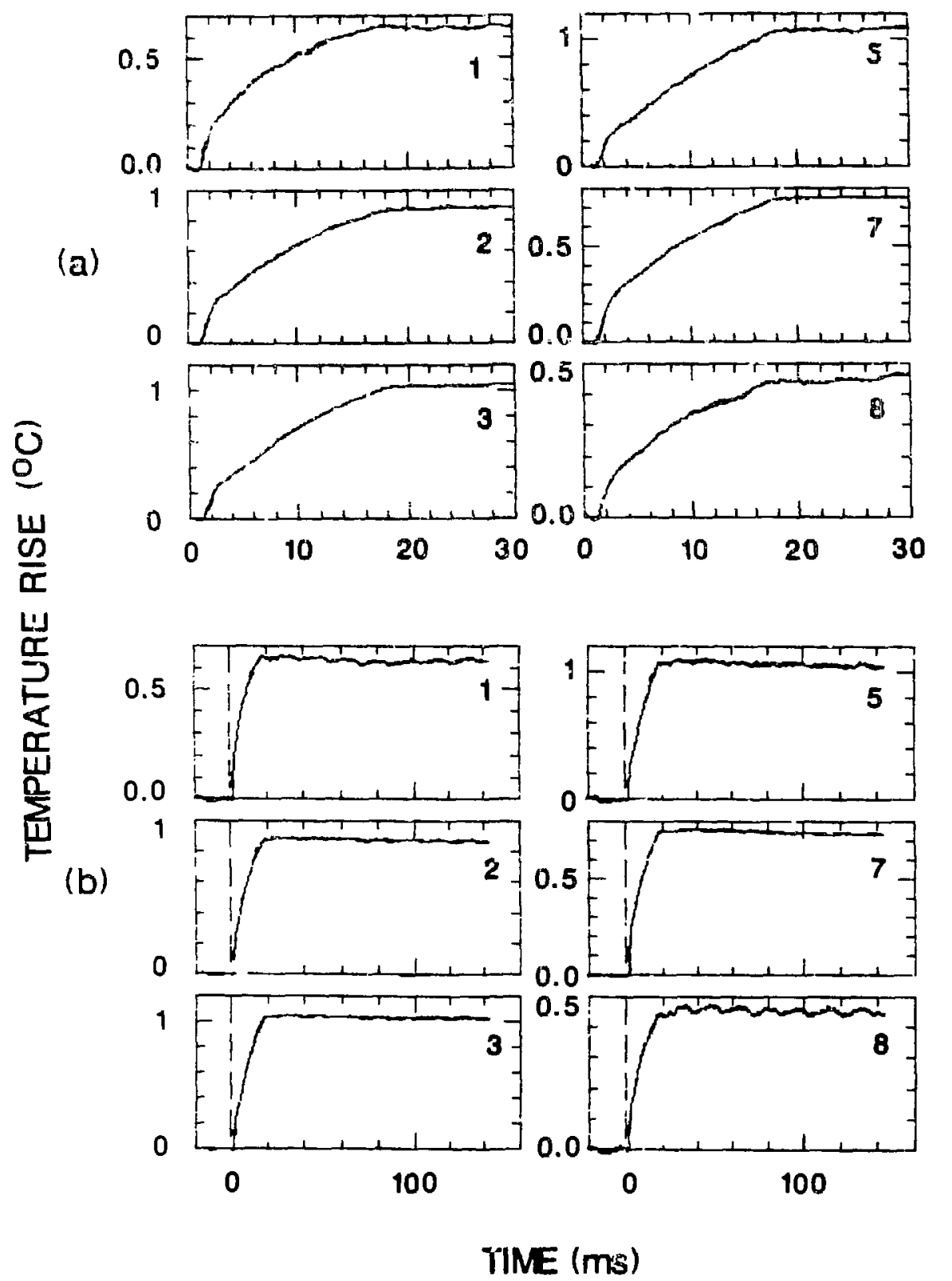

Fig. 8.

Part (a) shows bolometer raw data and part (b) the same data on a longer time scale. 


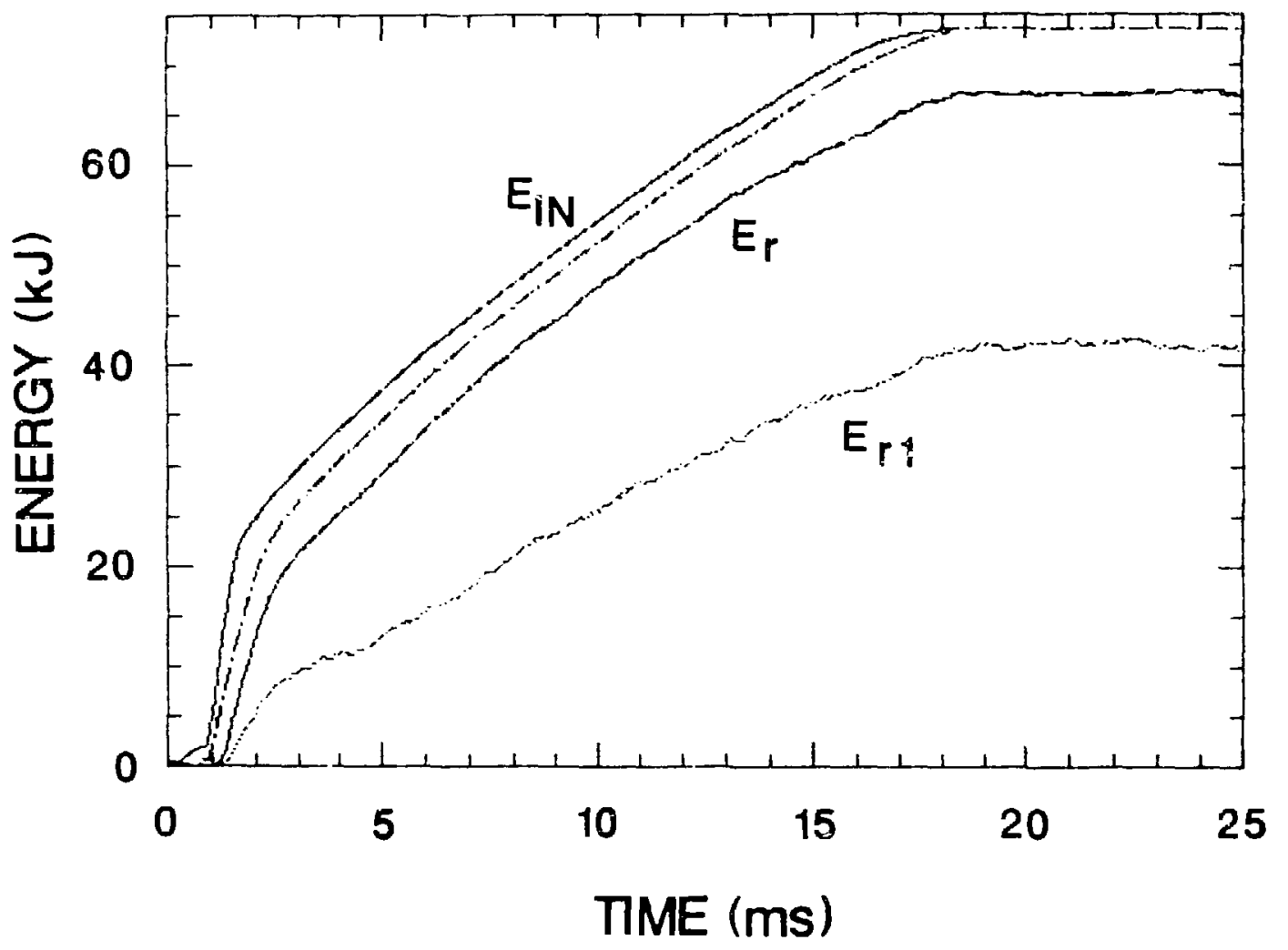

Fig. 9.

Radiated energies from bolometer data. This shot has almost $100 \%$ radiation losses. The upper curve is the total electrical energy input, the slightly lower dashed curve is the input energy minus the calculated magnetic field energy in the plasma (thus, approximately, the total energy lost by the plasma, assuming a small total plasma energy), and the two lower curves are the total radiated energy and the centrally radiated energy. 


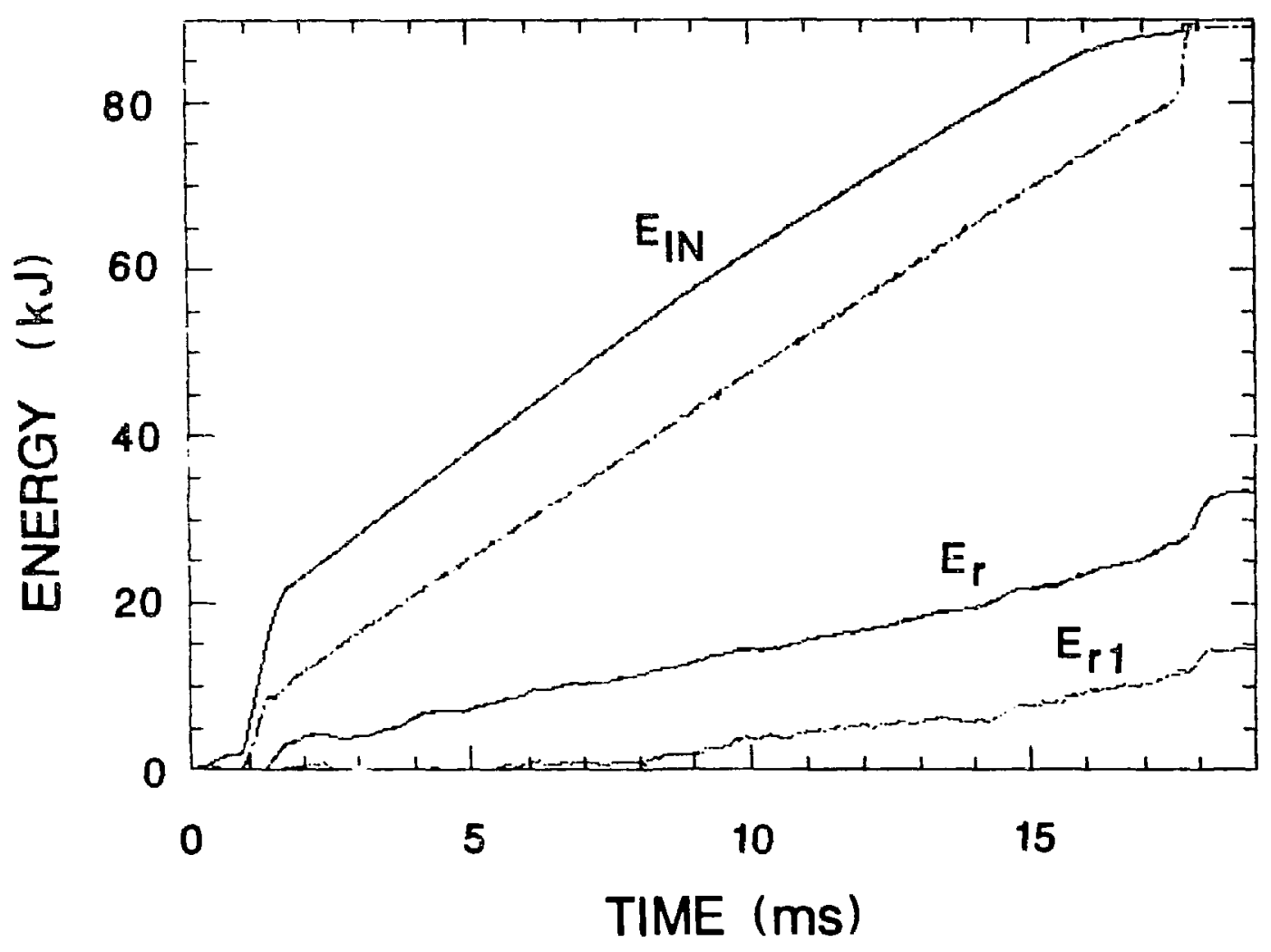

Fig. 10.

Radiated energies for the more usual case with the radiative fraction much less than 1 . Until about $8 \mathrm{~ms}$ the radiation is almost entirely from the edge region. 


\section{ACKNOWLEDGEMENT}

We wish to thank $\mathrm{J}$. Fernandez for informing us of the existence of a compilation of vacuum UV experimental data (Ref. 4).

\section{REFERENCES}

1. G. Millei, J.C. Iizgraham, and L.S. Schrank, Rev. Sci. Instrum. 53, 1410 (1982).

?. $\quad$. C. Ingraham and G. Miller, Rev. Sci. Instrum. 54, 673 (1983).

3. P. G. Carolan, et al., "New Results from HBTX-1A Reversed-Field Pinch," 10th International Conference on Plasma Physics and Controlled Nuclear Fusion Research (London, September 1984), Nucl. Fusion Supplement, Vol. 2, p. 449 (1985).

4. J. H. Weaver, C. Krafka, D. W. Lynch, and E. E. Koch, "Optical Properties of Metals" in Physik Daten 18 (1981).

5. G. Miller, Nucl. Fusion 24, 789 (1984). 